\title{
Violencia estudiantil contra docentes. Estudio de caso en una secundaria pública del estado de Jalisco
}

\section{Students' violence against teachers: a case study in a secondary school in Jalisco}

DOI: https://doi.org/10.32870/dse.v0i24.1049

\author{
Delia Patricia Pérez Márquez* \\ J. Isaac Uribe Alvarado** \\ Teresa Margarita Torres López ${ }^{* * *}$ \\ Roberto Oropeza Tena****
}

\begin{abstract}
Resumen
La violencia estudiantil contra docentes es una problemática que requiere particular atención en las discusiones respecto a la violencia escolar y en la generación de propuestas de intervención para erradicarla en los espacios educativos. En este trabajo se presentan resultados de un estudio de caso realizado en una secundaria pública de Jalisco, en donde se entrevistó a docentes y estudiantes. El objetivo fue conocer las formas de violencia que ejercen los estudiantes hacia docentes en secundaria, así como conocer las explicaciones sobre sus causas desde la perspectiva de los actores involucrados. Tras el análisis de los datos, se identificaron cuatro categorías de violencia hacia docentes: violencia verbal, violencia física, violencia patrimonial y violencia virtual. Se considera que los tipos de violencia pueden darse de forma directa e indirecta, y pueden infligirse desde lo individual o lo grupal. Las causas a las que se atribuye la violencia son distintas según la perspectiva de docentes y estudiantes; los y las docentes otorgan gran peso al contexto social y familiar del estudiante. Por su parte, los y las estudiantes mencionan como causantes las características de sus profesores. Se finaliza con una revisión a las repercusiones de la violencia estudiantil contra el personal docente y su impacto en el proceso educativo.
\end{abstract}

Palabras clave: Violencia escolar - violencia estudiantil hacia docentes - acoso al profesor - secundaria.

\section{Abstract}

Student violence against teachers is a phenomenon that requires special attention in the discussions about school violence and in the development of intervention proposals to eradicate violence in schools.

* Estudiante del Doctorado Interinstitucional en Psicología. Líneas de investigación: Psicología social de la educación, psicología educativa, autoregulación del aprendizaje. Universidad de Colima. México. delitricia@hotmail.com

** Doctor en Psicología. Líneas de investigación: Comportamientos de riesgo en grupos vulnerables. Juventudes y conducta sexual de riesgo. Universidad de Colima. México. iuribe@ucol.mx

*** Doctora en Antropología Social y Cultural. Líneas de investigación: Procesos psicológicos y antropológicos sociales. Universidad de Guadalajara. México.tere.torres.cucs@gmail.com

**** Doctor en Psicología. Líneas de investigación: Adicciones, tratamientos cognitivo-conductuales, enfermedades crónico-degenerativas, rendimiento académico. Universidad Michoacana de San Nicolás de Hidalgo. México. scherzo2112@yahoo.com 
This paper presents the results of a case study of a public middle school in Jalisco, where teachers and students were interviewed. The objective was to identify the forms of violence that students exert against teachers in middle school and to explore the causes attributed by students and teachers to explain this kind of violence. After our data analysis we created four categories: verbal violence, physical violence, property-related violence, and virtual violence. We noted that these types of violence can be direct or indirect and can be inflicted individually or by the group. The causes attributed to student violence against teachers differ depending on the perspective: teachers emphasize the students' family and context, while students refer to teacher's characteristics. Finally, some implications of student violence against teachers and their impact on the educational process are discussed.

Keywords: School violence - teacher targeted bullying - violence against teachers - middle school.

\section{Introducción}

La violencia escolar es un fenómeno que ha cobrado especial relevancia en las últimas décadas. Aunque el acoso entre pares es la problemática que ha mostrado mayor impacto, existen otras relaciones intraescolares que deben estudiarse para comprender a mayor profundidad la realidad educativa con el fin de propiciar el desarrollo de planes de intervención que mejoren la convivencia escolar.

En este estudio se aborda particularmente la violencia estudiantil hacia el docente. Esto, con el objetivo de conocer las formas de violencia que ejerce el estudiantado hacia los y las docentes en una secundaria en Jalisco, así como conocer las explicaciones sobre las causas de la violencia estudiantil hacia el personal docente, tanto desde la perspectiva de docentes como del alumnado.

\section{Antecedentes}

Se reconoce que el fenómeno de la violencia estudiantil hacia el docente no es nuevo, sin embargo, se considera un tema emergente; algunas revisiones documentales (Abril, 2019; Reddy et al., 2018) señalan que, a pesar del notorio incremento de estudios en la última década, estos siguen siendo insuficientes para comprender el fenónemo y sus implicaciones en el contexto educativo y social.

En México, una de las primeras aproximaciones fue un informe nacional realizado de manera conjunta por la Secretaría de Educación Pública y el Fondo de las Naciones Unidas para la Infancia (SEP / UNICEF, 2009) sobre la violencia de género en Educación Básica. Este documento puntualizó el fenómeno al incluir el rubro de agresiones hacia el profesorado como objeto de estudio. Se encontró que aproximadamente la mitad del personal docente participante afirmó haber sido agredido en los últimos dos años y señalaron como el principal agresor al estudiantado. Acerca del tipo de violencia recibida, la mayoría de participantes indicó la agresión verbal y el daño o robo de pertenencias, aunque pueden encontrarse otras formas de violencia que se 
consideran complementarias. Sin embargo, en años recientes han surgido publicaciones nacionales centradas en el fenómeno (Gómez, 2014; Gómez, Pérez, 2013, 2016; Valle-Barbosa et al., 2019), lo que habla de un creciente interés en explicar dicha problemática educativa y social.

El incremento de investigaciones sobre el tema también se refleja a nivel internacional (Abril, 2019; Reddy et al., 2018); en esta recopilación figuran estudios realizados en Colombia, Chile, Israel, Estados Unidos, China, España, Italia, Reino Unido y Sudáfrica. Fueron organizados siguiendo cuatro pautas según el abordaje del fenómeno: 1) Identificación de la violencia estudiantil contra docentes desde la perspectiva docente, 2 ) Identificación de la violencia estudiantil contra docentes desde la perspectiva de estudiantes, 3) Incidencia de las formas de violencia y 4) Consecuencias de la violencia. La totalidad de los estudios considerados en este apartado tienen como referencia el nivel secundaria o su correspondiente en otros países.

\section{Identificación de la violencia estudiantil contra docentes desde la perspectiva docente} Algunos estudios de corte cualitativo se han enfocado en identificar las formas de violencia estudiantil a través de la narrativa de los y las docentes. Gómez Nashiki (2014) analiza 25 entrevistas a profundidad aplicadas a docentes de secundaria que han sufrido algún tipo de violencia por parte de sus estudiantes a lo largo de su trayectoria. Señala que la forma de violencia más frecuente es la verbal, aunque figuran otras formas como la violencia física, el acoso, el desafío a la autoridad y el daño a bienes personales.

De manera similar, Galdames y Pezoa (2016) en Chile, realizaron grupos focales con profesores, con el fin de analizar los significados atribuidos a la violencia hacia los docentes. Además

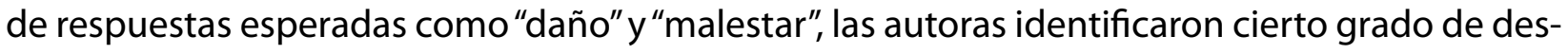
esperanza en los docentes cuando explican que la violencia forma parte del ecosistema escolar y hay que aprender a convivir con ella. Describen algunas conductas disruptivas por parte de estudiantes, consideradas no graves, y señalan el ámbito virtual como herramienta para violentar al docente.

Este último aspecto es abordado a detalle por Kyriacou y Zuin (2015), quienes analizan tres videos provenientes de países distintos (Brasil, Portugal e Inglaterra) para identificar la violencia virtual o ciberbullying hacia docentes, los cuales se hacen virales en sitios como Youtube. Los autores señalan la necesidad de desarrollar un nuevo marco conceptual desde el cual comprender el abuso estudiantil hacia sus docentes que incorpore los medios tecnológicos.

\section{Identificación de la violencia estudiantil contra docentes desde la perspectiva de estudiantes}

Los estudios que analizan el fenómeno desde la perspectiva estudiantil, tienen el objetivo de identificar las dinámicas relacionales entre los actores. Se caracterizan por obtener los datos mediante cuestionarios y su aplicación en amplias muestras de estudiantes a nivel secundaria. 
Estos estudios concluyen que los y las estudiantes perciben un proceso de reciprocidad entre el maltrato que reciben del profesorado y el que ejercen hacia él, lo que facilita el proceso de escalamiento de la agresión (Benbenishty et al., 2019; Domínguez et al., 2013; Mendoza, 2011). Particularmente, en el estudio de Valle-Barbosa et al. (2019) se estableció una prevalencia general de violencia de alumnos(as) hacia docentes de $41.9 \%$ y de docentes hacia alumnos(as) de $39.8 \%$, mostrando la equiparación de la violencia percibida.

\section{Incidencia de las formas de violencia estudiantil}

En estos estudios se obtuvieron los datos mediante cuestionarios en muestras amplias y se centran en la perspectiva del docente. Los criterios respecto a las conductas violentas que deben reportarse son diversas, por lo que los resultados son variados.

Los datos utilizados por Mcmahon et al. (2014) se basan en la encuesta nacional sobre violencia al docente a nivel primaria y secundaria en Estados Unidos; señalan que $80 \%$ de los docentes encuestados reportaron al menos una victimización en el espacio escolar y $94 \%$ de esos casos implicaban violencia perpetrada por estudiantes.

En el estudio de Morales et al. (2014) sólo se incluyeron conductas consideradas violentas moderadas y graves (no leves); las más señaladas, experimentadas durante el mes previo al estudio, fueron: maldiciones (39.9\%), robo de pertenencias (29.8\%), destrucción de pertenencias (17.9\%) y empujar (16\%).

Moon y McCluskey (2018) analizaron la formas de violencia sufridas por docentes durante el año previo al estudio, e indentificaron cinco tipos de acciones violentas: daño a la propiedad, violencia física, verbal, acoso sexual y agresión sin contacto. Las formas de violencia leve se reportaron con mayor frecuencia que las graves; el abuso verbal y la agresión sin contacto fueron las más señaladas. A pesar de que la violencia física y el acoso sexual fueron las menos frecuentes, aproximadamente uno de cada diez docentes lo reportaron.

\section{Consecuencias de la violencia estudiantil contra el docente}

Algunos estudios se han centrado en identificar y comprender las consecuencias de la violencia estudiantil hacia docentes mediante cuestionarios, considerando la perspectiva del docente. Analizan las consecuencias tanto en su desempeño profesional (Moon et al., 2015), por repercutir en la calidad de su trabajo y en las relaciones con sus estudiantes debido a los altos niveles de miedo generado; así como en relación con su salud mental (Woudstra et al., 2018) pues se encontró que las cuatro formas de violencia consideradas (verbal, física, indirecta y virtual) coinciden con altos niveles de ansiedad y depresión en docentes perpetrados. También se analiza la reacción institucional frente a los señalamientos de la violencia hacia el docente (Moon et al., 2019), donde se resalta la falta de seguimiento y medidas de protección o intervención para su resolución. 


\section{Conceptualización de la violencia}

En cuanto a la violencia como concepto, se utiliza la definición otorgada por la Organización Mundial de la Salud, instancia que la define como "el uso deliberado de fuerza física o el poder, ya sea en grado de amenaza o efectivo, contra uno mismo, otra persona o un grupo o comunidad, que cause o tenga muchas posibilidades de causar lesiones, muerte, daños psicológicos, trastornos del desarrollo o privaciones" (2003: 5).

Aunque las diversas investigaciones hacen referencia a distintas teorías para explicar la violencia, mantienen como relevante el análisis de este fenómeno desde una perspectiva ecológica. El Modelo Ecológico de Bronfenbrenner (1976) sugiere que los eventos de un ecosistema o entorno mayor influyen en la conducta de los individuos mediante su impacto en ecosistemas o entornos más pequeños, y de manera inversa. Es decir, que los múltiples entornos y las relaciones e interacciones en los entornos educativos de los y las docentes, así como las complicadas interacciones entre estos entornos, favorecen la explicación de la violencia desde una perspectiva más amplia y compleja, en donde se considera que la estructura educativa, las políticas públicas, la comunidad, las relaciones y las características de los individuos forman parte del fenómeno de violencia escolar dirigida a los docentes.

Por su complejidad y características, el enfoque ecológico se ha empleado para abordar la problemática de la violencia escolar y, particularmente, la violencia estudiantil hacia el docente (Reddy et al., 2018; Sorrentino, Farrington, 2019).

Desde esta perspectiva, se analizan los resultados del estudio, el cual tiene como objetivo principal conocer las formas de violencia que se presentan por parte de estudiantes hacia el personal docente en una secundaria de Jalisco, esto con la finalidad de categorizar y explorar el fenómeno.

\section{Método}

El estudio de caso desarrollado en este trabajo es de corte cualitativo y de tipo instrumental (Stake, 1995) ya que la institución educativa y los agentes que la componen son el medio por el cual se analiza un fenómeno específico: la violencia estudiantil hacia el docente. Se considera la prespectiva de Stake al identificar el estudio de caso como "el estudio de la particularidad y la complejidad de un caso, por el que se llega a comprender su actividad en circunstancias que son importantes" (1995: 7).

En este sentido, en este estudio es importante considerar dos antecedentes de la historia de este centro educativo. El primero es que el recinto tiene aproximadamente 35 años de haberse constituido y atiende su demanda educativa en dos turnos. No obstante haber sido conocido como una de las mejores escuelas secundarias, hace varios años se suscitó un evento particularmente relevante de violencia extrema, en el que una docente perdió la vida a manos de uno de sus estudiantes, quien dentro del aula y frente a sus compañeros la atacó con un arma de fuego. 
A varias décadas del hecho, algunos profesores mantienen el recuerdo y consideran que las implicaciones de este evento fueron un parteaguas en el proceso académico. Dicha institución modificó su dinámica tanto por el evento como por sus consecuencias; se menciona la existencia de un ambiente de tensión y miedo tanto de estudiantes como por docentes y dentro de la comunidad académica, que notoriamente ha incidido en la renuncia de varios docentes y el "repliegue" de otros, quienes evitaban confrontarse con estudiantes, generando laxitud en la disciplina y el proceso educativo durante los años que le siguieron. $Y$ un segundo elemento a considerar es que actualmente el plantel cuenta con pocos estudiantes matriculados, por lo que la disponibilidad de cupo implica la aceptación de estudiantes que han sido reubicados por problemas de conducta en otras instituciones.

Tomando en cuenta estos dos antecedentes, se consideró que la temática de la violencia estudiantil es sensible y algunas veces personal, por lo que se optó por un muestreo intencional. Los participantes fueron siete docentes y 19 alumnos(as) de los tres grados de secundaria (véase tabla 1). Las entrevistas con el personal docente se realizaron de manera individual, mientras las entrevistas a estudiantes se hiceron en grupos (Simons, 2011).

Tabla 1. Entrevistas y códigos

\begin{tabular}{|l|l|l|}
\hline \multicolumn{1}{|c|}{ Entrevista } & Código & $\begin{array}{l}\text { Grado de secundaria que } \\
\text { supervisa/imparte/cursa }\end{array}$ \\
\hline Entrevista a Docente 1 & ED1 & Múltiples \\
\hline Entrevista a Docente 2 & ED2 & Múltiples \\
\hline Entrevista a Docente 3 & ED3 & Múltiples \\
\hline Entrevista a Docente 4 & ED4 & Múltiples \\
\hline Entrevista a Docente 5 & ED5 & Múltiples \\
\hline Entrevista a Docente 6 & ED6 & Tercer grado \\
\hline Entrevista a Docente 7 & ED7 & Segundo grado \\
\hline Entrevista a Estudiante 1 & EE1 & Tercer grado \\
\hline Entrevista a Estudiantes 2 (4 estudiantes) & EE2 & Segundo grado \\
\hline Entrevista a Estudiantes 3 (2 estudiantes) & EE3 & Tercer grado \\
\hline Entrevista a Estudiantes 4 (6 estudiantes) & EE4 & Segundo grado \\
\hline Entrevista a Estudiantes 5 (4 estudiantes) & EE5 & Tercer grado \\
\hline Entrevista a Estudiantes 6 (2 estudiantes) & EE6 & Primer grado \\
\hline
\end{tabular}

Las entrevistas fueron de tipo semiestructurado con una función de entrevista conversacional (Simons, 2011). Se realizaron dentro del espacio educativo en momentos que los partici- 
pantes estuvieran disponibles y buscando espacios que permitieran la confidencia. La estructura de la entrevista abordó dos dimensiones: la percepción de violencia estudiantil hacia el docente dentro de su institución y las causas a las que se atribuye la violencia estudiantil contra el docente.

El ingreso a la escuela secundaria se realizó tras la autorización del director de la institución, mediante una petición escrita con información de la investigación y sus objetivos. La participación fue voluntaria y la totalidad de los participantes dieron su consentimiento para el uso de los datos con fines de investigación. Al inicio de cada entrevista se aclaró a los participantes el carácter confidencial de su participación y se les brindó la opción de no responder. Todas las entrevistas a estudiantes se llevaron a cabo dentro de la escuela, con el consentimiento de la autoridad educativa y cada uno de los participantes; también, acorde con la NOM-47-SSA-2105 para la atención del grupo etario de 10 a 19 años (específicamente en los artículos 3.18, 5.11, 5.14 y 6.8.1), la cual establece que es posible solicitar a un menor la firma de consentimiento informado directamente sin comunicarlo a los padres (NOM-047-SSA2-2015). La investigación se desarrolló a lo largo de un ciclo escolar, durante el turno verpertino.

Una vez transcritas la totalidad de las entrevistas, se realizó el procesamiento de la información, la revisión de contenidos y una lectura longitudinal. Posteriormente, se hizo reducción de los datos (Miles et al., 2018) mediante codificación y categorización, para exponerlos y concluir a partir de lo analizado; al final se hizo la verificación de los resultados mediante un proceso de triangulación (Carter et al., 2014).

Este proceso permitió la generación de cuatro categorías analíticas para la primera dimensión, la cual es referente a la percepción de la violencia estudiantil hacia el docente; dichas categorías fueron: $a$ ) violencia física, $b$ ) violencia verbal, c) violencia patrimonial y d) violencia virtual. Para la segunda dimensión, que hace referencia a las causas a las que se atribuye la violencia estudiantil hacia el docente, se obtuvieron tres categorías analíticas: $a$ ) la perspectiva docente en donde se considera a la escuela como reflejo de contextos violentos, $b$ ) perspectiva docente en donde se considera la escuela como generadora de violencia y c) perspectiva estudiantil, en donde se considera que la causa se relaciona con características de los y las docentes.

\section{Resultados}

\section{Manifestaciones de violencia estudiantil contra el docente}

Tras codificar las entrevistas y categorizar las formas de violencia estudiantil hacia los y las docentes, se identificaron cuatro tipos de violencia: violencia física, violencia verbal, violencia patrimonial y violencia virtual (tabla 2). Cada uno de estos tipos pueden observarse como acciones directas o indirectas, así como individuales o grupales. 
Tabla 2. Tipología de la violencia estudiantil hacia el docente

\begin{tabular}{|l|l|}
\hline \multicolumn{1}{|c|}{ Tipo de violencia } & \multicolumn{1}{c|}{ Definición } \\
\hline Violencia verbal & $\begin{array}{l}\text { El abuso verbal ocurre cuando algún estudiante usa el lenguaje, ya sea hablado } \\
\text { o escrito, para causar daño al docente. Esto incluye insultos, burlas, rumores, } \\
\text { apodos y menosprecios públicos para exhibir y ridiculizar. También se incluyen } \\
\text { las amenazas, sean públicas o privadas. }\end{array}$ \\
\hline Violencia física & $\begin{array}{l}\text { La violencia física ocurre cuando el/los estudiantes utilizan su cuerpo o algún } \\
\text { objeto para someter o controlar las acciones del docente. Por ejemplo: empujo- } \\
\text { nes, puñetazos, golpes, arrojarle objetos, etcétera. }\end{array}$ \\
\hline Violencia patrimonial & $\begin{array}{l}\text { La violencia patrimonial ocurre cuando el estudiante utiliza o da mal uso a los } \\
\text { recursos materiales de un docente sin su consentimiento. Por ejemplo, la des- } \\
\text { trucción de material o pertenencias. }\end{array}$ \\
\hline Violencia virtual & $\begin{array}{l}\text { Ocurre cuando se utilizan las plataformas virtuales y herramientas tecnológi- } \\
\text { cas, como las páginas de internet, los chats, blogs, redes sociales, correo elec- } \\
\text { trónico, mensajes de texto enviados por celular, foros, teléfono y otros medios } \\
\text { tecnológicos. }\end{array}$ \\
\hline
\end{tabular}

La totalidad de docentes y estudiantes entrevistados reconocieron una situación de violencia importante en la institución, haciendo especial referencia al acoso entre pares. Sobre la violencia ejercida por los estudiantes hacia los y las docentes, la mayoría aceptó su existencia dando ejemplos de situaciones cotidianas en los últimos años.

\section{Conductas disruptivas, la antesala de la violencia}

Previo a la descripción de los tipos de violencia estudiantil hacia docentes, es relevante mencionar de manera especial las conductas disruptivas y su relación con la violencia escolar.

Se trata de una serie de acciones estudiantiles que limitan el trabajo académico y el desarrollo de la clase, a la vez que muestran desinterés por el espacio áulico y el sistema escolar. Aquí se incluyen las acciones que son distintas a las indicaciones del docente y a la actividad que se desarrolla, así como hablar, discutir o distraer a compañeros y comportarse de forma indecuada durante las explicaciones del profesor. Galdames y Pezoa (2016: 162) incluyen acciones como "bostezar con exageración, hacer ruidos inapropiados, ignorar al profesor, o mantener un lenguaje no verbal desafiante".

Las conductas disruptivas que se desarrollan de forma aislada (sin relación con otras formas de violencia), se caracterizan por delimitarse en el tiempo y espacio de la clase y suelen estar dirigidas al proceso de enseñanza-aprendizaje, de manera que no suelen centrarse en el docente sino en la evasión del proceso de enseñanza-aprendizaje $y$, por lo tanto, inciden en el papel del docente como gestor del aprendizaje.

Si bien las conductas disruptivas no se consideran como un tipo de violencia por sí mismas al carecer de intencionalidad dañina hacia el docente, en relación con otras acciones es que pueden potenciar las formas de violencia estudiantil hacia el docente. Ya sea por generar con- 
flictos que pueden escalar a otras formas de violencia, en donde una reacción violenta evocaría otra aún más violenta, o por favorecer el mantenimiento de formas de violencia prexistentes que se reflejan en el espacio aúlico, al ser éste un espacio de convivencia obligado entre quien violenta y quien es violentado.

Jurado et al. (2020) describen las conductas disruptivas como una llamada de atención o un mecanismo de protesta por parte del estudiante, en relación con factores o situaciones que él no desea. Los autores señalan que el aburrimiento es una causante de las conductas disruptivas, particularmente en estudiantes que no tienen intención de continuar con sus estudios y no ven el sentido a permanecer en el aula o seguir los contenidos. Domínguez et al. (2013) indican que este tipo de acciones suelen ser las causas más frecuentes por las que se crean conflictos en el aula, ya sea por hablar o por comportarse de forma inadecuada durante las explicaciones del docente. Ochoa y Díez (2012) señalan que las conductas disruptivas son una de las causas que generan mayor tensión entre profesores y estudiantes y que pueden llevar a un conflicto interpersonal si no se afrontan y resuelven a tiempo.

Adicionalmente, el estudio de Jurado et al. (2020) indica que las conductas disruptivas están relacionadas estrechamente con la calidad de la relación que el estudiantado con sus profesores. Los y las estudiantes que consideran tener buena o muy buena relación con sus profesores muestran mucho menos conductas disruptivas $y$, por el contrario, quienes consideran que no existe una relación con su profesor, son quienes muestran un mayor número de conductas disruptivas, seguidos de quienes consideran que tienen una mala o regular relación.

Mendoza (2011) detectó que estudiantes violentos perciben un proceso de reciprocidad entre el maltrato que reciben y el que ejercen, lo que explica el escalamiento de la agresión. De forma similar, Benbenishty et al. (2019) señalan que los y las estudiantes que reportan haber violentado a sus docentes, también tienden a ser quienes reportan haber sido violentados(as) por ellos.

\section{Violencia verbal, uso del lenguaje como medio para violentar}

Ocurre cuando algún estudiante usa el lenguaje, ya sea hablado o escrito, para causar daño al docente; puede considerarse desde el uso de apodos, pasando por burlas e insultos o insinuaciones insultantes en referencia al físico o condiciones sociales, económicas, familiares y sexuales, hasta el uso de amenazas, ya sea de manera pública o privada.

Los señalamientos de este tipo de violencia son múltiples, por lo que figuran como la forma de violencia más común del estudiante hacia el docente. Los apodos y las burlas son una constante que suele minimizarse y normalizarse, al punto de considerarse parte de la experiencia educativa, tanto por estudiantes como por docentes.

Sin embargo, es importante considerar que el uso de lenguaje inapropiado, por sí solo, no 
constituye una manifestación de violencia al docente; lo es en la medida que se recurre a él para insultar, denigrar o intimidar. Incluso, cuando las expresiones señaladas se realizan sin conocimiento del docente pero se comparten con compañeros, son consideradas una forma indirecta de violencia. No obstante, cuando se comparte en un grupo y se expresa abiertamente al docente, se identifica como una situación de violencia verbal grupal.

En las entrevistas se describen eventos en los que de manera grupal y coordinada se burlan de las características físicas de un(a) docente y le caricaturizan de forma despectiva. Ya sea al decirlo de manera coral o al pegar en el aula dibujos y letreros que tengan el mensaje, o al coordinarse para reaccionar de alguna forma particular sin conocimiento del docente (como salirse del aula, esconderse o reírse tras cierto detonador acordado).

A continuación se integran las narraciones de dos eventos distintos, el primero en voz de una estudiante y el segundo narrado por un docente:

A la que le aventaron el lonche nos tocó como prefecta, nadie la quería, entonces pusieron dibujos de cerdos en todos los pintarrones y luego carteles de que no querían a la tronchatoro y luego le decían vieja gorda y así. Y la empezaron a ofender y empezaron a gritar con sus carteles en huelga. Eran los de primero, todos. Lo hicieron los del A y los B y también los del salón querían pero yo les dije que no. Es que si lo hacen unos ya es más fácil para todos los demás (Alumna, EE2).

Ya está bien, pero antes [la maestra] tenía un problema de bello facial. Hace un año, todo el grupo, imagínate al grupo cantándole a la maestra:"lobo lobito ¿estás ahí?" — ¡Cállense! "Lobo lobito ¿estás ahí? — ¡Que se callen! "Lobo lobito ¿estás ahí?" [Tras cada frase aumenta la intensidad del volumen]... Fue un acto de crueldad. Pero fue bien divertido. Nada que no estuviera ganado por la maestra. No lo justifico, pero sí lo disfruté (Profesor, ED2).

Específicamente en el tema de sexualidad, los y las estudiantes señalan que es común que se recurra a referencias sexuales para intimidar a docentes, ya sea acosando, juzgando o suponiendo sus preferencias o experiencias sexuales. Aunque en este estudio la violencia que se desarrolla en torno al acoso de índole sexual figura como violencia verbal y violencia física, en otros estudios se considera como otra categoría para la identificación de la violencia estudiantil hacia el docente (Patterson, Qiao, 2021). Las siguientes narraciones de los estudiantes ejemplifican esta forma de violencia:

Al psicólogo lo alburean mucho... hasta lo propios hombres. [...] él va pasando y le empiezan a decir que está bueno y él se pone rojo y se pasa... También al prefecto, pero porque es bien llevado. - “'A usted ya no se le para, porque está viejito" [y él responde] —“¿Quieres ver?, siéntate en ella". Es bien cochino (Alumna, EE6). 
La misma estudiante señala otra situación sobre este tema:

Es que [al profesor de educación física] le decían: es que usted me pone a hacer puras cosas de bailar, porque usted es gay. Ándele, déjeme jugar fútbol, es que es la verdad es muy gay que no se qué y lo hacen Ilorar y ya. Sólo así. Y sí los deja jugar fut (Alumna, EE6).

\section{Violencia física, la forma más directa y visible de la violencia}

Este tipo de violencia es la más visible, ocurre cuando el o la estudiante utiliza su cuerpo o algún objeto para someter o controlar las acciones del docente, ya sea mediante empujones, puñetazos, golpes, arrojar objetos, entre otras.

El rango de acciones que se consideran en este rubro van desde un tocamiento inapropiado, como palmadas en la espalda o saludo de manos con excesiva fuerza, pasando por arrojar objetos, tocamientos de índole sexual, golpes o hasta la violencia extrema que atenta contra la vida. Un profesor comenta al respecto:

He recibido agresiones físicas, las cuales pues, las tengo que contestar. Y ese tipo de cosas las he llevado frente a la autoridad, pero ahí quedan (Profesor, ED1).

En esta categoría también se consideran los empujones a modo de broma, los cuales son muy usuales. Este tipo de acciones, si bien tienen el componente físico, no corresponden a la actitud desafiante que la manifestación de violencia física directa suele poseer. Sin embargo, claramente corresponde a este rubro y el puntualizarlo permite su visibilización.

Sobre la forma indirecta de este tipo de violencia, se da cuando de manera "anónima" agreden al docente desde la distancia. Ya sea que se arrojen objetos con la intención de mofarse, humillar o lastimar a un docente o que otras personas lo ataquen físicamente por solicitud del estudiante. Los siguientes testimonios de los alumnos ejemplifican estas formas de violencia:

Sí me ha tocado ver que que les avienten cosas. Uno le aventó a la maestra un lonche en la cabeza [...] y otro un limonazo [risas], sí, y preguntaron que quién había sido y ya dijo que era él y ya se lo llevaron a la dirección y no pasó nada (Alumna, EE6).

Pues yo he visto que un compañero le avienta cosas a la maestra de español [...] está dictando y está hablando y él le avienta papeles y sí lo hace para atinarle [...] pero no pasa nada, le dice, te voy a llevar con el prefecto y ya (Alumno, EE4). 


\section{Violencia patrimonial, el daño a la propiedad privada}

Aunque menos documentada, la violencia patrimonial ocurre cuando el o la estudiante utiliza o hace mal uso de los recursos materiales de un(a) docente sin su consentimiento. El rango de situaciones, en función de gravedad, van desde ocultar o robar cosas del docente durante la clase hasta la destrucción de propiedad. En el último caso, se describe el daño al automóvil como el más común (Aguilera et al., 2007), que incluye ponchar llantas, rayar la pintura o quebrar los vidrios, pues usualmente se encuentra en un espacio próximo a la institución. Un docente con casi tres décadas de experiencia describe así su frustración al respecto:

Mi carro fue severamente dañado. Fue rayado con desarmador, quebraron retrovisores, poncharon llantas, rompieron cristales [...] nunca supe quién había sido, pero normalmente el motivo hacia cierto tipo de maestros como su servidor es la disciplina con la que tratamos a los muchachos, la firmeza con la que les hablamos (Profesor, ED3).

Una estudiante recuerda un evento de este tipo:

Una vez, a un maestro le rayaron el carro y le rompieron su vidrio [...] pues nomás, porque les cae mal (Alumna, EE3).

Por otra parte, el hurto de pertenencias es un evento que en ocasiones se utiliza a modo de broma. Sin embargo, el hecho de tomar las pertenencias de otra persona sin su consentimiento es un robo, lo cual se considera grave. Puede ser que el objeto se regrese después de algunas horas o días, sin embargo, la acción tiene una evidente manifestación de violencia. Domínguez et al., (2013) conciden con esta categoría de violencia estudiantil al docente y señalan acciones como: causar desperfectos en pertenencias, esconder pertenencias o material y robar al profesorado. McMahon et al. (2014) indican que más de la mitad de los maestros encuestados han sufrido daño a su propiedad. Algunos estudiantes mencionan eventos de este tipo:

En primero, hacían llorar casi casi al profesor. Porque era bien santito y le escondían sus cosas, sus lentes se los quitaban y los ponían en sus butacas o en sus cajitas [...] a veces yo abogaba por el profe porque ay, a veces yo si sentía feo, son muy manchados y no se lo merece, es muy "pan blandito" para nosotros. Él nos hablaba todavía bien, pues, ya muchachos por favor dénmelo y así, mis compañeros se reían, yo se los regresaba (Alumna, EE6).

A un profe le escondían los marcadores para que no pudiera dar la clase. Ah, y a una maestra le robaron su cartera con todo y dinero. Y no, no los corrieron. Bueno, a Tony creo que sí lo corrieron (Alumno, EE4). 
Aguilera et al. (2007) señalan que, si bien no es la causa única, el daño a los objetos de los profesores es una manera de reaccionar por parte de los alumnos(as) ante la imposibilidad de hacerlo directamente con las personas.

\section{Violencia virtual, fenómeno emergente y en desarrollo}

La violencia virtual ocurre cuando se utilizan las plataformas virtuales y herramientas tecnológicas, como las páginas de internet, los chats, blogs, redes sociales, correo electrónico, mensajes de texto enviados por celular, foros, teléfono y otros medios tecnológicos para dañar al docente.

Durante las entrevistas surgieron diversos eventos que se caracterizan de esta forma; se hace mención de algunos de los más inusuales, considerando el uso del teléfono celular, el correo electrónico y las redes sociales.

En el semestre previo a la investigación, alumnas de la institución utilizaron la comunicación móvil para amenazar a su maestro; mediante mensajes y llamadas amenzaban de muerte al docente y a su familia. De forma similar, pero empleando el correo electrónico, otro docente señala que estudiantes se apropiaron de su cuenta de correo y mandaron amenazas a sus contactos. Aunque se trataba de una "broma", según palabras de estudiantes involucrados, provocó malestar en la familia y en el propio docente.

Con mensajes a mi celular, correo electrónico, llegaron a invadir mi correo electrónico. Me decían "te vas a morir". A la hora que entraron, provocaron el caos con los contactos, de también sentirse amenazados, entonces provocaron un movimiento grande al grado de llegar al seno familiar. Como mis contactos eran mis hermanos, pues me decían ¿Qué va a pasar? ¿Qué estás haciendo? ¿En qué estás metido? [...] me gritaban "te vas a morir y cuida a tu hijo porque se va a morir, ya sabemos por dónde vas y que carro traes (Profesor, ED1).

Una estudiante también describe el espisodio:

[Cuando le llamaban] le mentaban la madre, y todo ese tipo de cosas así, le dijeron muchas ofensas de su familia. Lo amenazaron de que se iba a morir, de que le iban a hacer algo a su carro, el maestro se molestó, le iba a poner una demanda, le quitaron el celular, la iban a correr. A su mamá también le iban a poner una demanda, a las otras que participaron también le iban a poner demanda y todo eso, entonces nada más a las otras les pusieron demanda y a ésta de nuestro salón, le pusieron una advertencia nada más. Y de ahí para acá nos tienen más checaditos. [...] Después el maestro no la quería ni voltear a ver, como ahorita está dándole clase pues como que ya se la lleva mejorcillo. Ella era muy trabajadora en primero, pero se empezó a juntar con otras amistades que le llevaron a otras cosas y fue perdiendo eso y ahorita no ha venido en dos semanas... (Alumna, EE1). 
También durante el estudio se detectó el uso de las redes sociales por parte de estudiantes para la exhibición y mofa de docentes. Se encuentran comentarios en los perfiles de estudiantes refiriéndose a sus maestros, e incluso se gestionan espacios grupales y abiertos a exalumnos(as), en donde la temática central es la institución escolar, por lo que los y las docentes figuran frecuentemente. En este espacio es tolerado el uso de apodos y expresiones groseras en torno a ellos, en ocasiones se incluyen fotos y videos que pudieran haber sido tomados sin su consentimiento.

Otros estudios (Galdames, Pezoa, 2016: 163) señalan el ámbito virtual como herramienta que puede usarse de forma eficaz"para humillar y denostar la imagen del docente, a partir de sus características físicas, sus hábitos, su manera de hablar o vestir"; por una parte, la virtualidad posibilita el anonimato, y por otra, permite una difusión inmediata y masiva. Se señalan bromas que se graban o fotografían, para posteriormente publicarlas. Domínguez et al. (2013) señalan acciones específicas como: publicar comentarios en redes sociales de ofensa, insultos o amenazas al profesorado, grabar o hacer fotos en el celular para burlarse y publicar en internet fotos/ videos ofensivos. Kyriacou y Zuin (2015) indican que el cyberbullying implica un giro radical respecto a la forma en que los estudiantes retan la autoridad de sus docentes, haciendo uso de medios digitales.

\section{Las formas de la violencia y la interacción entre violencias}

Cabe resaltar que, si bien se pretende hacer una distinción clara de las categorías de violencia estudiantil hacia el docente, muchas veces las acciones corresponden a dos o más categorías. A su vez, cada uno de estos tipos puede ser directo o indirecto. Mientras en el primero la víctima es la receptora de la acción y el victimario es el ejecutor, en las segundas se recibe el daño de manera no directa o por medio de terceros (anónimamente), como cuando se utilizan otros espacios para intermediar la acción, por ejemplo, los chismes, el hurto, las acciones grupales que excluyen al sujeto, entre otros aspectos.

La evidencia indica que las manifestaciones violentas varían mucho en cuanto a las formas. Si bien pueden presentarse por parte de un solo estudiante en un espacio público o privado, también es posible que suceda de manera grupal y puede darse en cualquiera de las tipologías mencionadas.

Gómez Nashiki (2014) menciona al respecto que las manifestaciones de violencia hacia el docente no siempre implican una disrupción en el orden establecido de manera inmediata, sino que crecen y gradualmente aumentan la intensidad, adoptando distintas formas y combinaciones. 


\section{Causas de la violencia estudiantil contra el docente desde la perspectiva docente}

Al preguntar a los y las docentes a qué atribuyen la existencia de la violencia ejercida por estudiantes y dirigida a docentes, se identificaron tres pautas centrales. En la primera, se habla de la frustración que los jóvenes experimentan al encontrarse con carencias afectivas en casa y en la escuela. Los y las docentes atribuyen la violencia estudiantil a la ausencia de madurez emocional en los y las estudiantes y, a su vez, mencionan la incapacidad del profesorado para afrontar y contener estas situaciones de índole psicológica.

Por otra parte, los criterios se dividen en función del rol de la escuela respecto a los actos de violencia que surgen dentro de ella. En el primero se menciona a la escuela como espacio en donde se reflejan los contextos violentos, y en el segundo, la escuela se considera un espacio generador de violencia.

\section{Escuela como reflejo de contextos violentos}

En primera instancia, consideran el ambiente externo en el cual los jóvenes viven y conviven como el núcleo del problema, ya sea por cuestiones familiares, las amistades que frecuentan o la situación de la comunidad-barrio donde viven; se considera que son espacios generadores de diversas "frustraciones" en personas jóvenes, quienes eligen la escuela como lugar para desahogarlas. En este aspecto, se identifican tres argumentos centrales:

- Las familias disfuncionales. Señalan que los y las adolescentes conviven con violencia en casa, en ocasiones sin un tutor que se haga responsable de ellos o que sea capaz de establecer hábitos y límites. También mencionan la inexistencia de actividades extracurriculares o tiempo de calidad que se acuerden como familia. Los siguientes fragmentos tomados de entrevistas con docentes lo ejemplifican:

Generalmente, quien es vulnerable o violento es porque lo ven en la casa, pues entonces esas personalidades son de los adultos. Y que antes había al menos la presencia de un adulto en la casa y ahorita los papás son complacientes porque no están y lo suplen con cosas, pero los seres humanos necesitamos una atención integral, una atención cercana (Profesora, ED5).

Todos absorben los problemas de los adultos, aquí tenemos, la mayoría de los niños son hijos "ping-pong", entonces, un tiempo están con la mamá y otro tiempo están con el papá, andan botando, los papás separados. La otra situación es que son muchos niños del mismo barrio, y si es un barrio problemático, pues se trae la problemática a la escuela (Profesor, ED2).

Entonces aquí tenemos que no hay valores, no saben qué es un valor porque en su casa no lo viven. Si tú te fijas, a la redonda no hay un área verde en donde ellos puedan jugar, no hay uni- 
dades deportivas o si vas a jugar, todo te cuesta. Todo te cuesta y no traemos dinero, entonces el chavo ¿a dónde se va? A la calle (Profesor, ED1).

- Falta de conciencia sobre la función de la escuela. Se indica que los padres ven la escuela como un tipo de guardería, porque los jóvenes no tienen una ocupación en casa y se omite la función de la familia como responsable primordial de la educación y la trasmisión de valores.

La escuela, los papás la usan como refugio, como guardería y el alumno piensa que viene a ser cuidado. $Y$ entonces no lo toman como una continuación de la educación y eso provoca que se desprestigie la educación (Profesor, ED1).

Yo pienso que eso viene de casa, que los padres no están muy involucrados, muy preocupados por darles esos cuidados y esa responsabilidad que les tienen que dar. Y mandan a los hijos a que la escuela los eduque y la educación se recibe en casa, no en la escuela. Aquí se da, se dan conocimientos, se imparten conocimientos, pero la educación es de casa, no es de la escuela. Entonces sí, si son más irreverentes, si son groseros, les ponen apodos a los maestros, pero, bueno, pues a mí, a uno, le corresponde que ellos tengan respeto por sus maestros (Profesora, ED4).

En su momento la escuela era un bastión que formaba conducta y modelos de conducta, el día de hoy parece ser que es el refugio de muchachos desocupados que no tienen conciencia de a lo que vienen, los papás tampoco tienen mucha conciencia, ehh... y se han forjado esta serie de fenómenos que convierten a la escuela en un laboratorio de formación experimental de violencia. Desde la violencia psicológica hasta la violencia física, de la violencia más imperceptible, persuasiva, hasta la violencia química con las drogas (Profesor, ED3).

- Desinterés por los procesos educativos. Los padres no muestran interés por las actividades escolares, ni dan seguimiento al desarrollo de sus hijos e hijas. En ocasiones por la edad o por cuestiones laborales, pero algunos docentes consideran que no se cuenta con el apoyo de la familia en la educación de los hijos. Algunos maestros mencionan que si se les llama para notificarles una falta del estudiante, los padres, en lugar de colaborar a la educación de los adolescentes, solapan sus decisiones y actitudes.

Y es lo que aquí vemos en la escuela, que de diez alumnos sólo tienes bien a dos niños que tienen a su familia completa y vienen a la escuela bien, comen bien, hacen deporte, se divierten, y el $80 \%$ no. No conocen un cine, no conocen un teatro, entonces tú tratas de inculcarles algo cultural... y se viene abajo (Profesor, ED1). 
Yo antes le hablaba al papá y venía, ahora ya no [...] les dices a las señoras y te dicen que no, no te creen. Hasta que ya se esperan a la salida y lo ven llegando y todavía así solapan la actitud (Profesora, ED5).

\section{La escuela como generadora de violencia}

El segundo aspecto considerado, y recurrente como explicación de las manifestaciones de violencia en los jóvenes, se centra en la escuela; se la describe como un espacio en donde se generan relaciones de violencia. Es decir, que las causas se atribuyen las relaciones interescolares. Se habla de la institución que carece de orden, del personal docente que se desvincula de su labor intencionalmente y de docentes que no logran posicionarse como autoridad.

- Falta de rigurosidad en la dirección y normatividad. Se menciona que la ausencia de una autoridad educativa firme y constante favorece el crecimiento de la violencia en la institución, así como el hecho de que no existan protocolos o normativas sobre cómo manejar los casos de violencia y, particularmente, la que se ejerce contra el docente.

- Desvinculación emocional en docentes. Algunos docentes señalaron la exigencia hacia la docencia en cuestiones administrativas y académicas; se les imponen rígidos objetivos por cumplir que se reflejan en la exigencia a sus estudiantes. De ellos y ellas requieren que se entregue la lección, un trabajo o un proyecto terminal; cuando esto no sucede, hay una reacción de molestia por parte del docente. Sin embargo, se indica que muchos de esos estudiantes están en la clase sin desearlo, e incluso, realizan algunas de las actividades sólo para complacer al docente; si de cualquier forma reciben rechazo por parte del docente, su reacción posiblemente se incline hacia la violencia.

Muchas veces, en la atención en el grupo los maestros no nos damos el tiempo de individualizar al sujeto y de hacer un acercamiento un poquito más humano, lo seguimos viendo como un número de la lista, seguimos viéndolos como sujetos que tienen que entregar un trabajo, un proyecto terminal, entregar la lección, etc. Y si no, pues entonces me molesto porque no avanzó, porque no hizo, etc... y ese ciclo interminable. Los muchachos hacen lo que no quieren hacer. Muchos de ellos vienen a la escuela a hacer lo que no quieren hacer, pero lo hacen en busca de que alguien les dé ese afecto, ese cariño, esa pasión; y bueno, cuando la respuesta es una sanción, es una negativa, es un rechazo, pues entonces estalla el fenómeno de la violencia (Profesor, ED3).

- Incapacidad en docentes para marcar límites. Los y las docentes indican que el alumnado no tiene malas intenciones al cometer las acciones que se consideran violentas, por lo que señalan 
que cuando suceden actos de violencia es responsabilidad del docente que no supo suprimir la violencia; o que es su responsabilidad al permitir que los y las estudiantes sean irrespetuosos con su persona o con su proceso de enseñanza. Otros docentes, en cambio, señalaron que también se da porque los y las docentes son muy rígidos, que imponen disciplina en sus estudiantes y estos les amenazan para que disminuyan el nivel de exigencia.

Yo considero que cuando se han dado casos en que un alumno enfrenta a un maestro, es porque el maestro no tuvo la habilidad como maestro de suprimir la violencia hacia el alumno que el alumno hacia el maestro y de eso estoy convencidísimo. “Es que el muchacho" - El muchacho te esté esperando para decirte algo (Profesor, ED2).

Ahh, sí. Pero porque yo peco de hacer de más y de no saberme callar [...] Si tú eres una persona barquito, nomás te meces a donde te lleva la marea y ahí nunca vas a tener problemas (Profesor, ED1).

Digamos que los muchachos agarran sus barquitos maestros, ¿no? Y hay maestros que hasta se les suben al escritorio y le bailan arriba, y hay maestros como el maestro y su servidor que... inmediatamente ponemos la disciplina (Profesor, ED3).

Yo soy de las que les dice "no les digas así, es tu maestro" y les hablan por su nombre [...] pero, bueno, hay maestros que les encanta que les falten al respeto, entonces ya es... sí, que les gusta que les digan por su nombre, que les hablen de "wey" y que... pues ya cada quién ¿no? (Profesora, ED7).

Entonces, ¿por qué agredo al otro? porque yo estoy siendo agredido y busco a una persona débil y la ataco, para eso también tiene que haber personas vulnerables y que lo permitan por no tener seguridad en sí mismo. El que no tiene bases en casa para poder poner un límite. Si un maestro permite ser agredido ¿qué está pasando con él? (Profesora, ED6).

\section{Perspectiva del alumnado}

Para los y las estudiantes, las explicaciones a estas interacciones tienen una visión muy distinta. Según los datos obtenidos en las entrevistas a estudiantes, ni el contexto ni los medios tienen relevancia. Señalan primordialmente las actitudes de los y las docentes como la causa que les hace merecedores de las respuestas violentas por parte de sus estudiantes.

Sólo uno de los 19 entrevistados señaló el trato en casa como un factor importante en la relación estudiante-maestro y menciona la presión entre pares, asegurando que las acciones violentas hacia sus profesores se realizan para "quedar bien" con sus amigos y amigas. El resto 
de los testimonios se refiere a actitudes de los y las docentes por las que les "caen gordos", mencionan que son injustos, muestran favoritismo, discriminan por aspectos físicos o culturales, que siguen regañando por actos pasados o sin conocer las circunstancias reales, muestran poca tolerancia o no tienen interés sobre la clase que imparten.

También señalan, con especial énfasis, el trato que se da a alumnos(as) dóciles y a alumnos(as) desmadrozos(as). Algunos profesores sólo explican y atienden a algunos grupos de estudiantes que siguen las indicaciones y realizan las tareas, sin embargo, a los que consideran que se ven "cholos" o que ya tienen antecedentes de haber reprobado, los ignoran o no les explican aunque se lo soliciten directamente. Incluso mencionan casos en que, sin razón aparente, algunos docentes son groseros con algún(a) estudiante en particular. A continuación un diálogo entre un grupo de estudiantes:

E1: Haz de cuenta, cuando ven un cholillo, ellos dicen "no, no hay que hacer caso". Y lo discriminan. Y así.

E2: O cuando son desmadrozos no los quieren. No les hacen caso, no le explican.

E3: Agreden a los maestros porque ellos los ignoran. Y ya que les hacen caso es nada más para regañarlos.

E1:Yo últimamente ya iba a entregar mi trabajo, pero ya no me lo recibió.

E2: Es que hay niñas que son favoritas. Hay una [respuesta] que le decía él [señala a E1] y cuando la niña la dijo, a ella sí le hizo caso y a él no.

E4: Ay, es que ya no pueden hacer nada porque luego luego, ya no les dejan hacer nada. De todo los regañan.

E2: Ese maestro se agarra a la prefecta y se la pasa diciéndole cosas de nosotros. En primero habíamos varios que no traíamos el material a tiempo y de eso se agarraba y nos empezaban a reprobar en todo. Tuve que hacerle tres trabajos extras y otros nomás hacían uno y se los revisaba. O las niñas no traían material, hasta el siguiente mes y no les decía nada...

Explican como injusto que algunos profesores abusen de su posición de autoridad para hacer sentir mal a alumnos(as) que les caen mal. Algunos estudiantes hablaron de cómo se han sentido humillados o han observado situaciones en donde los y las docentes son abusivos con estudiantes y los hacen llorar en el aula. En estos casos, los y las estudiantes consideran las acciones violentas dirigidas al docente como una forma de defenderse de estos ataques. Dos testimonios de estudiantes señalan estos aspectos:

Que a veces [los maestros] se sienten que son autoridad y se sienten con el derecho de hacer sentir mal porque les caen mal. A veces a los maestros ya les caen mal los alumnos. Como a ella, Sandybell [señala a su compañera de clase], ella le preguntó “¿Por qué ahora trae estos 
pantalones?"... Porque siempre los trae entubados. Y él le contestó que de ella todo estaba feo, "hasta la cara la tienes fea", pero ella le hizo una pregunta bien. Y él dijo "Ay, lo tuyo todo es feo" le dijo de las calcetas y de todo. Sólo a ella la trata así y es el único maestro. Desde que llegó la trae contra ella (EE3).

E1: Porque algunos te humillan.

E2: A mí, si me dicen algo, yo les reclamo. Si ni mi mamá me grita, ni mi papá ni me pega, ni me gritan... ¿por qué ellos sí?

E1: Pues sí, te humillan de que te hacen menos en las clases, diciéndote de cosas feas y hacerte llorar [...] es que a veces dicen "es que tú para qué estás aquí, mejor vete a tu casa, tú no deberías de haber nacido", así... y yo así de que... [cara de sorpresa].

E2: Como igual no estudies, ¿edá?

E1: Pues sí, es que teníamos clase en ese salón [señala un aula], estábamos trabajando y platicando porque yo le estaba explicando a una compañera y dijo: ¿otra vez platicando?"le dije:"no, es que le estoy explicando" y mi compañera dijo: "Sí, me está explicando" y me dijo: "Ay sí, ahora eres muy lista, ¿no?", y le dije "aunque no me crea" y me dice: "te cambias para acá y te volteas a la pared” y ya me cambié y me dijo: ¿OOtra vez? No m’ija, es que ¿para qué estás aquí?, tú no deberías de haber nacido. ¿Para qué estás aquí? a ver dime, estás aquí y nomás causas problemas". Nomás que yo soy así, muy tímida. Pero ya después mi hermano, porque mis papás no sabían, pues ya le dije y quería venir a decirle y le dije que no... y ya después de cualquier cosa que me decía, yo le decía y le digo “¡No me diga eso!". Sé que me veo grosera a veces por cómo contesto, pero es necesario, para defenderme (EE6).

De manera similar, varios alumnos(as) afirman que no deben dejarse del docente y por eso contestan en el mismo tono en el que reciben la interacción. Señalan que las acciones de otros estudiantes por defenderse del maestro o maestra les han permitido no ser molestados por estos. Incluso, se dice que los y las estudiantes que molestan al docente gozan de beneficios adicionales: les dejan salir del aula y no les exigen las actividades que exigen al resto.

\section{Conclusiones}

Los datos de las entrevistas a los distintos actores nos muestran que las acciones estudiantiles violentas hacia docentes son normalizadas. Lo que inicialmente se señala como no existente, se convierte en una serie de situaciones de violencia cotidiana durante las clases y fuera de ellas.

La mayoría del personal docente niega o minimiza el problema, particularmente en relación con su experiencia personal, aunque sí pueden mencionar múltiples casos que 
involucren a compañeros. El siguiente fragmento muestra la respuesta de una docente que fue señalada como violentada por estudiantes:

No, no... no violentos. Son irr... irreverentes. Sí se ha perdido el respeto, pero yo creo que es generacional. Antes eras "el maestro" ahora ya no eres "el maestro", ahora les vale y te mientan tu madre y te dicen cosas, pero son cosas que ya pasa en todos lados (Profesora, ED4).

En otros estudios del tema se muestra una tendencia similar, la indencia de violencia hacia docentes es más alta cuando se declara sobre otros(as) docentes y no sobre sí mismos(as). Los datos señalan que si el reporte de conductas violentas son sobre las observadas en otro docente en lugar de experimentadas, las cifras casi se duplican (Morales et al., 2014).

También coincide con los resultados obtenidos por Gómez (2016), quien considera que los y las docentes niegan violencia directa hacia su persona porque al aceptarlo vulneran su estatus como profesional. En la misma línea, Abril (2019) señala que uno de los motivos por los cuales la violencia hacia docentes aún es un tema tabú, podría deberse a que asumirlo implicaría perder credibilidad profesional y personal, imposibilitándolo responder a los procesos académicos y sociales que demanda un grupo de estudiantes.

Sin duda, se trata de una problemática poco reconocida, no sólo por lo estudiosos de los ambientes educativos sino por lo propios agentes que conviven en las escuelas, quienes consideran las continuas conductas violentas de estudiantes como parte "natural" de las dinámicas intraescolares o como una falla en el docente que no puede mantener un adecuado control grupal y, por lo tanto, carece de las competencias necesarias para ejercer su profesión. Es así como la violencia hacia el docente se vuelve una problemática silente, en donde los maestros no están dispuestos a admitir la violencia recibida por parte de sus estudiantes.

Los y las estudiantes en su totalidad pueden explicar las acciones que realizan en contra de los docentes y no tienen dificultades en señalarlos públicamente. Sin embargo, prefieren no admitir su responsabilidad directa. Cuando se les señala, acusan a alguien más o mencionan a los otros involucrados. Es decir, que tanto docentes como estudiantes pueden hablar de la problemática, siempre y cuando no los implique de forma directa.

La violencia en el espacio educativo, además de limitar las posibilidades de un adecuado proceso de enseñanza-aprendizaje, tiene repercusiones significativas en los individuos que la reciben. Se conocen consecuencias de la violencia contra el docente a nivel profesional, física y emocional; posiblemente la primera es la más evidente, y se vincula con consecuencias emocionales.

Se ha encontrado que la violencia estudiantil hacia el docente tiene como consecuencia emociones negativas como inseguridad, miedo, soledad, enojo e impotencia, que perjudican 
el desarrollo de su trabajo (Gómez, 2014), además de desempoderamiento, baja moral y baja motivación para ejecutar los roles que le son asignados en el sistema educativo (Woudstra et al., 2018).

Se señala que no es necesario que los eventos violentos sean catalogados como graves para tener un efecto negativo en el docente, el abuso verbal y el físico sin contacto son los factores más comunes en el desarrollo del estrés y el malestar docente (Moon et al., 2015).

Además, la relación entre docentes y estudiantes resulta afectada. Profesores(as) han señalado mantener altos niveles de miedo y desconfianza tras ser victimizados (Moon, McCluskey, 2018), lo que implica una reducida capacidad de neutralizar otros tipos de violencia escolar.

A nivel personal, se ha encontrado que este tipo de eventos tiene repercusiones que van más allá del espacio institucional. Además del estrés, ser docentes receptores de violencia estudantil se vincula con altos niveles de ansiedad y depresión, sin importar el tipo de violencia que hayan recibido (Woudstra et al., 2018).

Acciones críticas como amenazas del tipo "si me reprueba, la mato" y la intimidación por medio de amenazas de índole física o sexual, se consideran agravios verbales que no dañan al docente, sin considerar que en dichos casos el docente está obligado a mantener una relación con quien le agrede.

En este estudio, los estudiantes explican cómo es que, tras acciones violentas hacia sus docentes, a los perpetradores no les "pasa nada", incluso si es del conocimiento del director, quien en ocasiones podía solicitarles que "sean respetuosos con sus maestros", pero sin intervenir de otra forma, lo que estudiantes describen como desinterés del director por lo que sucede en la escuela y una invitación a no preocuparse por las consecuencias de la violencia hacia los y las docentes. En otras ocasiones, incluso, removieron de su puesto al docente violentado por petición de los estudiantes que de manera grupal le acosaban verbalmente.

En los casos que se llevaron ante las autoridades, se señala que el director opta por crear acuerdos entre las partes, reduciendo las sanciones que les corresponden a ambos o aplicando las sanciones correspondientes, las cuales, no obstante, implican movimientos mínimos dentro de las dinámicas cotidianas; también es posible que involucre a los padres con apercibimientos verbales, aunque preferentemente se mantienen intramuros.

Los profesores que admitieron haber sufrido algún tipo de violencia señalaron, que los estudiantes involucrados no modificaron su actitud tras manejar el conflicto a nivel institucional, por el contrario, se mostraban amenazantes señalando la desventaja del docente, lo que provoca un mayor malestar.

Se identifica que el apoyo institucional que se otorga cuando se reporta algún caso de violencia es inexistente (Gómez, 2014) o resulta ineficiente e inadecuado (Moon et al., 2019). Abril (2019) señala que institucionalmente se impide que los casos de maltrato hacia docentes tengan el tratamiento que les corresponde. Se prefiere minimizarlo y arreglarlo de forma interna. 
Y por otra parte, existe malestar por parte de docentes al percibir que tras reportar la situación de forma institucional, los y las estudiantes involucrados no viven las consecuencias ni cambian su actitud (Moon et al., 2019).

Estos datos nos muestran cómo el sistema educativo tiene elementos que favorecen la violencia en sus diferentes formas. De acuerdo con la teoría de Bronfenbrenner (1976), los eventos del entorno escolar, en este caso la violencia estudiantil a profesores, podrían ser efecto de la interrelación de las personas con otros entornos con los que también interactúan. La familia, la comunidad, la cultura y el mismo espacio educativo en donde las personas se desarrollan, influyen en la manera en que interactúan con los otros. Por tanto, es necesario analizar cómo se entiende y conforma la violencia en dichos espacios para favorecer la comprensión de las interacciones educativas.

Se sugiere continuar las investigaciones en torno al fenómeno de la violencia escolar, particularmente el de la violencia estudiantil dirigida al docente, y considerar su existencia en las discusiones sobre la violencia escolar y en el diseño de acciones e intervenciones para la eradicación de la violencia en contextos educativos.

\section{Referencias}

Abril, C. (2019). Malestar docente y violencia escolar, una relación por definir: revisión documental de la década del noventa a la actualidad. Revista Logos, Ciencia y Tecnología, 12(1), 188-202. https://doi.org/10.22335/rlct.v12i1.1045

Aguilera, M.; G. Muñoz; A. Orozco (2007). Disciplina, violencia y consumo de sustancias en escuelas primarias y secundarias de México. México: INEE. http://www2.sepdf.gob.mx/equidad/comunidad escolar/directivos/planeacion/herramien ta/disciplina.pdf

Benbenishty, R.; R. Astor; V. López; M. Bilbao; P. Ascorra (2019). Victimization of Teachers by Students in Israel and in Chile and its Relations with Teachers' Victimization of Students. Aggressive Behavior, 45(2), 107-119. https://doi.org/10.1002/ab.21791

Bronfenbrenner, U. (1976). The Experimental Ecology of Education. Educational Researcher, 5(9), 5-15.

Carter, N.; D. Bryant-Lukosius; A. Dicenso; J. Blythe; A. Neville (2014). The Use of Triangulation in Qualitative Research. Oncology Nursing Forum, 41(5), 545-547. https://doi.org/10.1188/14. ONF.545-547

Domínguez, J.; A. López; M. Pino; E. Álvarez (2013). Violencia escolar: la díada interpersonal profesorado-alumnado. European Journal of Investigation in Health, Psychology and Education, 3(2), 75. https://doi.org/10.30552/ejihpe.v3i2.46

Galdames, A.; C. Pezoa (2016). Violencias hacia profesores de Enseñanza Media en la región me- 
tropolitana de Chile. Revista Rumbos TS. Un Espacio Crítico para la Reflexión en Ciencias Sociales, (14), 155-172. http://revistafacso.ucentral.cl/index.php/rumbos/article/view/40

Gómez, A. (2014). La violencia de alumnos hacia maestros en escuelas secundarias de Colima, México. Pensamiento Educativo: Revista de Investigación Educacional Latinoamericana, 51(2), 19-34. https://doi.org/10.7764/pel.51.2.2014.2

Gómez, A. (2016) Violencia de alumnos hacia docentes, la otra cara de la violencia escolar. Modelos educativos: ¿cómo ir en otra dirección? (88-99). México: Universidad Autónoma de Nayarit. Jurado, P.; A. Lafuente; M. Justiniano (2020). Conductas disruptivas en educación secundaria obligatoria: análisis de factores intervinientes. Contextos Educativos. Revista de Educación, 25(25), 219-236. https://doi.org/10.18172/con.3827

Kyriacou, Ch.; A. Zuin (2015) Cyberbullying of Teachers by Students on YouTube: Challenging the Image of Teacher Authority in the Digital Age. Research Papers in Education, 31(3), 255-273. https://doli.org/10.1080/02671522.2015.1037337

Mcmahon, S.; A. Martínez; D. Espelage; C. Rose; L. Reddy; K. Lane; E. Anderman; C. Reynolds; A. Jones; V. Brown (2014). Violence Directed against Teachers: Results from a National Survey. Psychol. Schs., (51), 753-766. https://doi.org/10.1002/pits.21777

Mendoza, B. (2011). Bullying entre pares y el escalamiento de agresión en la relación profesor-alumno. Psicología Iberoamericana, 19(1), 58-71. http://www.redalyc.org/articulo. oa?id=133920896007

Miles, M.; A. Huberman; J. Saldaña (2018). Qualitative Data Analysis: A Methods Sourcebook. Sage publications.

Morales, M.; J. Álvarez; A. Ayala; P. Ascorra; M. Bilbao; C. Carrasco; V. Lopez; D. Olavarría; S. Ortiz; C. Urbina; B. Villalobos (2014). Violencia escolar a profesores: Conductas de victimización reportadas por docentes de Enseñanza Básica. Revista de Estudios Cotidianos, 2(2), 91-116. https://dialnet.unirioja.es/servlet/articulo?codigo=5118372\&info=resumen\&idioma=SPA

Moon, B.; M. Morash; J. Jang; S. Jeong (2015). Violence Against Teachers in South Korea: Negative Consequences and Factors Leading to Emotional Distress. Violence and Victims; New York, 30(2), 279-292.

Moon, B.; J. McCluskey (2018). An Exploratory Study of Violence and Aggression Against Teachers in Middle and High Schools: Prevalence, Predictors, and Negative Consequences. Journal of School Violence, 19(2), 122-137. https://doi.org/10.1080/15388220.2018.1540010

Moon, B.; M. Morash; J. McCluskey (2019). Student Violence Directed Against Teachers: Victimized Teachers' Reports to School Officials and Satisfaction with School Responses. Journal of Interpersonal Violence. https://doi.org/10.1177/0886260519825883

NOM-047-SSA2-2015 (2015), Para la atención a la salud del grupo etario de 10 a 19 años de edad. México: Secretaría de Gobernación. 
Ochoa, A.; E. Díez-Martínez (2012). La escuela como sistema social de convivencia y su relación con algunos problemas de violencia. La percepción de los estudiantes de primaria y secundaria. Diálogos sobre Educación, (4). http://www.dialogossobreeducacion.cucsh.udg.mx/ index.php/DSE/article/view/363

Organización Mundial de la Salud (2003). Informe mundial sobre la violencia y la salud. Ginebra: CEPAL, sobre la base de la OMS. https://iris.paho.org/bitstream/handle/10665.2/725/9275315884.pdf

Pérez, D. (2013). Reflexiones en torno a la violencia de género hacia las docentes de educación básica. Revista de Estudios de Género. La Ventana, IV(38), 405-409. https://doi.org/10.32870/ Iv.v4i38.501

Qiao, B.; M. Patterson (2021). Teachers as Targets of Student Bullying: Data from China and The United States. Psychology in the Schools. https://doi.org/10.1002/pits.22493

Reddy, L.; D. Espelage; E. Anderman; J. Kanrich; S. McMahon (2018). Addressing Violence Against Educators through Measurement and Research. Aggression and Violent Behavior, (42), 9-28. https://doi.org/10.1016/j.avb.2018.06.006

Sanmartín, J.; R. Gutiérrez; J. Martínez; J. Vera (2010). Reflexiones sobre violencia. México: Editorial Siglo XXI.

Secretaría de Educación Pública (SEP); Fondo de las Naciones Unidas para la Infancia (UNICEF) (2009). Informe nacional sobre violencia de género en Educación Básica en México. México: SEP.

Simons, H. (2011). El estudio de caso: Teoría y práctica. Madrid: Ediciones Morata.

Sorrentino, A.; D. Farrington (2019). Individual, Family, Peer, and School Risk Factors for Teacher Victimization. Educational Sciences: Theory and Practice, 19(4), 1-13. https://doi. org/10.12738/estp.2019.4.001

Stake, R. (1995). The Art of Case Study Research. Sage publications.

Valle-Barbosa, M.; A. Muñoz; R. Robles-Bañuelos; M. Vega; M. Flores-Villavicencio; G. GonzálezPérez (2019). La violencia y acoso escolar en una escuela de Guadalajara, México. Revista Iberoamericana de Educación, 79(2), 43-58. https://doi.org/10.35362/rie7923180

Woudstra, M.; E. Van Rensburg; M. Visser; J. Jordaan (2018). Learner-to-Teacher Bullying as a Potential Factor Influencing Teachers' Mental Health. South African Journal of Education, 38(1). https://doi.org/10.15700/saje.v38n1a1358 